\title{
RICCI CURVATURE AND HOLOMORPHIC CONVEXITY IN KÄHLER MANIFOLDS
}

\author{
KAREN R. PINNEY
}

(Communicated by Jonathan M. Rosenberg)

\begin{abstract}
In this paper we show that if a smoothly bounded, relatively compact domain in a complex manifold admits a complete Kähler metric with certain bounds on its Ricci tensor, then the domain must be holomorphically convex. This gives an obstruction for the existence of a complete Kähler-Einstein metric on such domains.
\end{abstract}

\section{INTRODUCTION}

The existence of a complete Kähler-Einstein metric imposes some strong analytic conditions on an open manifold. Mok and Yau obtained a very satisfying result on the relationship between Kähler-Einstein metrics and holomorphic convexity for a bounded Riemann domain $\Omega$ [6]. They proved that the following are equivalent:

(1) $\Omega$ is a domain of holomorphy (holomorphically convex).

(2) $\Omega$ admits a complete Kähler-Einstein metric of negative Ricci curvature.

(3) $\Omega$ admits a complete Hermitian metric with Ricci curvature nonpositive and bounded below.

The paper of Mok and Yau completes and extends earlier work of Cheng and Yau [3].

In this paper we first give two examples which demonstrate that the existence of a Kähler-Einstein metric on an open manifold does not in general imply holomorphic convexity. We then state and prove Theorem 1, which says that for a smoothly bounded, relatively compact domain in a complex manifold, holomorphic convexity is necessary for the existence of a complete Kähler metric with certain bounds on its Ricci tensor.

\section{OPEN KÄHLER-EINSTEIN MANIFOLDS}

WHICH ARE NOT HOLOMORPHICALLY CONVEX

First, let $X=\mathbb{C}^{2} / \Lambda$, where $\Lambda$ is the group generated by $(1,0),(0,1)$, and $(a, b)$ over $\mathbb{Z}$. Then $X$ inherits a complete Ricci-flat metric from $\mathbb{C}^{2}$.

Received by the editors December 20,1990 and, in revised form, November 5, 1992.

1991 Mathematics Subject Classification. Primary 53C55, 32C10; Secondary 53C25, 32E10. 
However, if $a$ and $b$ are complex numbers such that $1, a$, and $b$ are linearly independent over $\mathbb{Z}$, then $X$ is not holomorphically convex (see [10]).

One may also construct an example with negative Ricci curvature: let $X=$ $\mathbb{B}^{n} / \Gamma$, where $\mathbb{B}^{n}$ is the unit ball with Poincaré metric in $\mathbb{C}^{n}$ and $\Gamma$ is an arithmetically defined discontinuous group of isometries of $\mathbb{B}^{n}$. Then $X$ inherits a complete Kähler-Einstein metric of negative Ricci curvature from $\mathbb{B}^{n}$. The Satake-Baily-Borel compactification of $X$ is a normal analytic space $\bar{X}$ obtained from $X$ by adjoining a finite number of points (see [1]). If $n \geq 2$, then the normality of $\bar{X}$ implies that holomorphic functions on $X$ extend to $\bar{X}$ and therefore are constant, so $X$ is not holomorphically convex. Because $X$ is not a bounded Riemann domain, this does not contradict the result of Mok and Yau.

This second example differs markedly from the domains considered by Cheng, Mok, and Yau in that the Kähler-Einstein metric on $X$ has finite volume. The complete Kähler-Einstein metric on a bounded Riemann domain of holomorphy has infinite volume; in fact, the coefficient of the volume form grows at least as fast as $d^{-2}(-\log d)^{-2}[3,6]$.

We note that Mok and Zhong have generalized the Satake-Baily-Borel result; they showed that, under a mild topological condition, a complete Kähler manifold with finite volume, negative Ricci curvature, and bounded sectional curvatures is biholomorphic to a quasi-projective manifold [7]. See [5, 8, 9, 13, 16].

\section{Notation}

Henceforth $\Omega$ will denote a relatively compact domain in a complex manifold $M$. We denote the volume form associated to a Hermitian metric $g$ by $V_{g}$. For a volume form $V$ which equals $\left(\frac{i}{2}\right)^{n} v(z) d z_{1} \wedge d \bar{z}_{1} \wedge \cdots \wedge d z_{n} \wedge d \bar{z}_{n}$ in local coordinates, the Ricci form $\operatorname{Ric}(V)$ is the globally defined $(1,1)$-form $\frac{i}{2} \partial \bar{\partial} \log v$.

\section{PRELIMINARY OBSERVATIONS}

The existence of a complete Kähler metric on $\Omega$ with bounds on its Ricci form immediately imposes some analytic conditions on $\Omega$. A theorem of Ohsawa says that a smoothly $\left(C^{1}\right)$ bounded domain which carries a complete Kähler metric must have pseudoconvex boundary [12]. It need not, however, be holomorphically convex [4]. If $\Omega$ admits a Hermitian metric $g$ and a volume form $V$ such that $\operatorname{Ric}\left(V_{g}\right)<\operatorname{Ric}(V)\left(\operatorname{resp} . \operatorname{Ric}\left(V_{g}\right)>\operatorname{Ric}(V)\right)$, then $\Omega$ carries a strictly plurisubharmonic function $\log \left(V_{g} / V\right)$ (resp. $\left.\log \left(V / V_{g}\right)\right)$. In particular, $\Omega$ may not contain any compact analytic set. For example, if $V$ is the volume form of a Ricci flat metric on $M$ and $\Omega$ admits a metric of negative Ricci curvature, then $\Omega$ must admit a strictly plurisubharmonic function.

\section{STATEMENT OF THE THEOREM}

Theorem 1. Let $M$ be a complex manifold and $\Omega \Subset M$ a relatively compact domain with $C^{2}$ boundary. Suppose that $\Omega$ admits a complete Kähler metric $g$ whose Ricci curvature is bounded below and such that

(*) there is a neighborhood $T$ of $\partial \Omega$ in $M$ and a volume form $V_{M}$ on $T$ 
such that, for some positive definite $(1,1)$-form $\omega$ defined on $T$,

$$
\operatorname{Ric}\left(V_{M}\right)-\operatorname{Ric}\left(V_{g}\right)>\omega
$$

on $T \cap \Omega$.

Then $\Omega$ is holomorphically convex. If $V_{M}$ is defined on a neighborhood of $\bar{\Omega}$ and $(*)$ holds on all of $\Omega$, then $\Omega$ is Stein.

This theorem very nearly proves a conjecture of Narasimhan. He conjectured that if $D$ is a domain with smooth boundary in a projective manifold $M$, if $c_{1}(M) \geq 0$, and if $D$ supports a complete Kähler metric with Ricci curvature bounded between negative constants, then $D$ is Stein. The above theorem proves this conjecture if one adds to Narasimhan's conjecture the hypothesis that locally near $\partial D$ the complete Kähler metric dominates a multiple of a Hermitian metric on $M$.

\section{TECHNICAL LEMMA}

We first prove a technical lemma. Let $X$ be a complete Riemannian manifold. Fix $p \in X$, and let $S_{k}=\{x \in X: d(x, p) \geq k\}$, where $d(x, p)$ denotes the distance from $x$ to $p$. Let $f$ be a continuous real-valued function on $S_{k_{0}}$. Let $\Gamma_{k}$ denote the graph of $f$ over $S_{k}, k \geq k_{0} . \Gamma_{k}$ is a closed submanifold (with boundary) of $\mathbb{R} \times X$. Let $\sigma: \mathbb{Z}^{+} \rightarrow \mathbb{Z}^{+}$be strictly increasing, and let $p_{\sigma(k)}$ denote the point $(\sigma(k), p)$ in $\mathbb{R} \times X$. Because $\Gamma_{k}$ is closed and $\mathbb{R} \times X$ is complete with respect to the product metric, there is a geodesic segment $g_{k}$ in $\mathbb{R} \times X$ from $p_{\sigma(k)}$ to $\left(f\left(q_{k}\right), q_{k}\right) \in \Gamma_{k}$ whose length is the distance from $p_{\sigma(k)}$ to $\Gamma_{k}$.

Lemma 1. One of the following is true:

(a) There is some $\sigma$ such that $q_{k}$ may be chosen in the interior $\stackrel{\circ}{S}_{k}$ of $S_{k}$ for all $k$ large enough.

(b) There is a point $q \in X$ such that if $d(x, p)>d(q, p)$, then $f(x) \leq$ $f(q)$.

Proof of Lemma 1. Suppose (a) is false. Then there is some $k$ such that $q_{k}$ must be on $\partial S_{k}$ for all $\sigma(k)$. Since $\partial S_{k}$ is compact, $f$ achieves its maximum on $\partial S_{k}$ at some point which we denote by $q$. Now

$$
\begin{aligned}
d\left(p_{\sigma(k)},\left(f\left(q_{k}\right), q_{k}\right)\right) & =\sqrt{\left(\sigma(k)-f\left(q_{k}\right)\right)^{2}+d\left(p, q_{k}\right)^{2}} \\
& =\sqrt{\left(\sigma(k)-f\left(q_{k}\right)\right)^{2}+k^{2}},
\end{aligned}
$$

which is minimized when $\left(\sigma(k)-f\left(q_{k}\right)\right)^{2}$ is minimized. Therefore, one may choose $q_{k}=q$ for all $\sigma$ such that $\sigma(k) \geq f(q)$. Suppose there were a point $x \in \stackrel{\circ}{S}_{k}$ such that $d(x, p)>k$ and $f(x)>f(q)$. Since $d\left(p_{\sigma(k)},(f(x), x)\right)=$ $\sqrt{(\sigma(k)-f(x))^{2}+d(x, p)^{2}}$ and $d\left(p_{\sigma(k)},(f(q), q)\right)=\sqrt{(\sigma(k)-f(q))^{2}+k^{2}}$, the inequality

$$
d\left(p_{\sigma(k)},(f(x), x)\right)<d\left(p_{\sigma(k)},(f(q), q)\right)
$$

is equivalent to the inequality

$$
f(x)^{2}-f(q)^{2}+d(x, p)^{2}-k^{2}<2 \sigma(k)(f(x)-f(q)) .
$$


Inequality (1) is true for $\sigma(k)$ large enough; this contradicts the assumption that $q_{k}$ must be on $\partial S_{k}$ for all $\sigma$. Therefore, $f(x) \leq f(q)$ for all $x$ such that $d(x, p)>k$, which proves $(\mathrm{b})$.

\section{PROOF OF THE THEOREM}

For $z \in \Omega$, let $d(z)$ denote the distance from $z$ to $\partial \Omega$, measured in a fixed metric on $M$, shrinking $T$ if necessary so that $d$ is $C^{2}$ on $T \cap \Omega$. Because $\Omega$ carries a complete Kähler metric, it must have pseudoconvex boundary. Thus $i \partial \bar{\partial}(-\log d(z))$ is bounded below near $\partial \Omega$ (see, e.g., [14]). For $k$ large enough,

$$
\tilde{\phi}(z)=k \log \frac{V_{g}}{V_{M}}-\log d(z)
$$

is strictly plurisubharmonic in $T \cap \Omega$. We extend $\tilde{\phi}$ to a continuous plurisubharmonic function $\phi$ which equals $\tilde{\phi}$ on $T^{\prime} \cap \Omega, T^{\prime}$ a relatively compact neighborhood of $\partial \Omega$ in $T$. To show that $\Omega$ is holomorphically convex, we need only show that $\phi$ is an exhaustion function, that is, that $\{z \in \Omega: \phi(z) \leq a\}$ is compact for all real $a$ (see [11]). Clearly $\phi$ is an exhaustion function if $V_{g} / V_{M}$ is bounded away from zero.

To show that this is the case, we cover $\partial \Omega$ by coordinate balls $B_{\alpha}$ with Poincare metric $g_{\alpha}$ and associated volume form $V_{\alpha}$. We compare $V_{g}$ to $V_{\alpha}$ on $B_{\alpha}^{\prime} \cap \Omega$, where $B_{\alpha}^{\prime} \Subset B_{\alpha}$ and $\partial \Omega \subset \bigcup_{\alpha} B_{\alpha}^{\prime}$. In [6], Mok and Yau prove the following Schwarz lemma for volume forms:

Lemma 2. Let $X$ be a complete Kähler manifold with Ricci curvature bounded below and let $Y$ be a complex manifold of the same dimension with a volume form $V_{Y}$ such that $\operatorname{Ric}\left(V_{Y}\right)$ is negative definite and $\left(-\operatorname{Ric}\left(V_{Y}\right)\right)^{n} \geq c V_{Y}, c>0$. Suppose $f: X \rightarrow Y$ is a holomorphic map and the Jacobian is nonvanishing at one point. Then $\left(f^{*} V_{Y}\right) / V_{X}$ is bounded above on $X$.

We would like to apply this theorem to our situation, with $X=Y=B_{\alpha}^{\prime} \cap \Omega$, $V_{X}=V_{g}, V_{Y}=V_{\alpha}$, and $f=$ identity. However, we may not apply the lemma directly because the metric $g$ is not complete on $B_{\alpha}^{\prime} \cap \Omega$. In proving their Schwarz lemma, Mok and Yau use the completeness of $g$ only to apply Yau's Maximum Principle (see [15]). Because $g$ is "complete near $\partial \Omega$ ", we may adapt the proof as follows.

Let $W=\bigcup_{\alpha} B_{\alpha}^{\prime}$. Define $u(x)=\min _{\alpha, x \in B_{\alpha}^{\prime}}\left(V_{\alpha} / V_{g}\right)$. The function $u$ is positive and continuous on $W$. Since $V_{\alpha}$ and $V_{M}$ are continuous on $B_{\alpha}$, they are comparable on $B_{\alpha}^{\prime} \cap \Omega$, so it suffices to show that $\overline{\lim }_{x \rightarrow \partial \Omega} u(x)$ is finite. Apply Lemma 1 with $X=(\Omega, g)$ and $f=-u^{-1 / 2 n}$. If (b) of Lemma 1 is true, we are done; then $\overline{\lim }_{x \rightarrow \partial \Omega} u(x) \geq(u(q))^{-2 n}$. We therefore suppose that (a) is true. From Mok and Yau's proof of the Schwarz lemma for volume forms we obtain

$$
\triangle\left(u^{-\frac{1}{2 n}}\right) \leq \frac{-1}{2 n} u^{-\frac{1}{2 n}}\left(R+n u^{\frac{1}{n}}\right)+\frac{2 n+1}{(2 n)^{2}} u^{-\frac{1}{2 n}-2}|\nabla u|^{2}
$$

for all points at which $u$ is smooth. Here $\triangle$ denotes the Laplacian with respect to $g$ and $R$ the scalar curvature of $g$. Suppose $u$ is smooth at an infinite number of the points $q_{k}$. Then using (a) and Yau's proof of his Maximum 
Principle, we obtain a sequence $\left\{q_{k}^{\prime}\right\} \subset W$ such that

$$
\begin{gathered}
\lim _{k \rightarrow \infty}-\left(u\left(q_{k}^{\prime}\right)\right)^{-\frac{1}{2 n}}=\varlimsup_{x \rightarrow \partial \Omega}-u(x)^{-\frac{1}{2 n}}, \\
\left|\nabla\left(u\left(q_{k}^{\prime}\right)\right)^{-\frac{1}{2 n}}\right|<\frac{1}{k} \\
-\triangle\left(u\left(q_{k}^{\prime}\right)\right)^{-\frac{1}{2 n}}<\frac{1}{k} .
\end{gathered}
$$

Multiplying (2) by $u^{-\frac{1}{2 n}}$ and applying (4) and (5) yields

$$
\left.-\frac{1}{k}\left(u\left(q_{k}^{\prime}\right)\right)^{-\frac{1}{2 n}}<\frac{-1}{2 n}\left(u\left(q_{k}^{\prime}\right)\right)^{-\frac{1}{n}}\left(R\left(q_{k}^{\prime}\right)+n\left(u\left(q_{k}^{\prime}\right)\right)^{\frac{1}{n}}\right)\right)+\frac{2 n+1}{k} .
$$

Let $-c_{1}$ be a lower bound for $R$. Then

$$
-\frac{1}{k}\left(u\left(q_{k}^{\prime}\right)\right)^{-\frac{1}{2 n}}<\frac{c_{1}}{2 n}\left(u\left(q_{k}^{\prime}\right)\right)^{-\frac{1}{n}}-\frac{1}{2}+\frac{2 n+1}{k} .
$$

Equation (3) implies $\lim _{k \rightarrow \infty}\left(u\left(q_{k}^{\prime}\right)\right)=\overline{\lim }_{x \rightarrow \partial \Omega} u(x)$. We assume $\overline{\lim } u>0$; if not, we are done. As $k \rightarrow \infty$, the left-hand side of (6) approaches 0 and the right-hand side approaches $\frac{c_{1}}{2 n}\left(\varlimsup_{\lim } u\right)^{-\frac{1}{n}}-\frac{1}{2}$, so $\varlimsup_{x \rightarrow \partial \Omega} u(x)<c_{1}^{n} n^{-n}$.

If the points $q_{k}$ must be chosen so that $u$ is smooth at only a finite number of them, we still obtain a sequence $\left\{q_{k}^{\prime}\right\}$ such that (3) holds, but we have neither the inequality (2) nor the estimates (4) and (5). Let $S$ be the set of points in $\partial \Omega$ which are limit points of the points at which $u$ is not smooth. Suppose that $\varlimsup_{x \rightarrow \partial \Omega} u(x)$ is infinite. If $y \in \partial \Omega \backslash S$, then $\varlimsup_{x \rightarrow y} u(x)$ is finite; otherwise, $q_{k}^{\prime}$ could be chosen in the set on which $u$ is smooth. Now shift the balls $B_{\alpha}$ slightly to obtain a new covering $\left\{\tilde{B}_{\alpha}\right\}$ of $\partial \Omega$ so that the function $\tilde{u}$, which is constructed analogously to $u$, is smooth near all points of $S$. We obtain $\left\{\tilde{q}_{k}^{\prime}\right\}$ as before. Because $\overline{\lim }_{x \rightarrow y} \tilde{u}(x)$ is infinite if and only if $\overline{\lim }_{x \rightarrow y} u(x)$ is infinite, we see that $\tilde{u}$ is smooth at an infinite number of the points $\tilde{q}_{k}^{\prime}$. The previous argument then shows that $\overline{\lim }_{x \rightarrow \partial \Omega} \tilde{u}(x)$ is finite. Therefore, $\overline{\lim }_{x \rightarrow \partial \Omega} u(x)$ is finite.

Under the additional condition that $V_{M}$ is defined on a neighborhood of $\bar{\Omega}$ and ( $*$ ) holds on all of $\Omega$, the function $\tilde{\phi}$ is defined on all of $\Omega$ and is a continuous strictly plurisubharmonic exhaustion function for $k$ large enough, so we conclude that $\Omega$ is Stein.

Remark. Mok and Yau used some similar techniques to show that given certain conditions on Ricci curvature, an increasing union of Stein subdomains in a Kähler manifold is Stein [6].

\section{AN EXAMPLe of Grauert}

This theorem may be applied to an example of Grauert. In [4], he constructs several examples of domains with pseudoconvex boundary in a complex manifold which are not holomorphically convex. One of these is a pseudoconvex neighborhood $U$ of the image of the zero section in a particular complex line bundle $F$ over a complex manifold. In this example the total space $F$ carries a Kähler metric with vanishing Ricci curvature (see [2]). The image of the zero section is a compact analytic set in $U$, so, by our earlier observations, $U$ admits no Hermitian metric of strictly negative Ricci curvature, in particular, no Kähler-Einstein metric of negative Ricci curvature. 
Grauert shows that $U$ supports no nonconstant holomorphic functions. By Theorem 1, $U$ admits no complete Kähler metric whose Ricci curvature is bounded below and whose Ricci tensor near $\partial U$ is negative and bounded away from zero. In fact, in this particular example we may locally compare $V_{g}$ to the volume form of a complete metric on a polydisc rather than to $V_{\alpha}$, showing that $\log \left(V_{g} / V_{F}\right)$ is an exhaustion function. We therefore see that $U$ may support no complete Kähler metric whose Ricci curvature is negative near $\partial U$ and bounded below.

\section{ACKNOWLEDGMENT}

I would like to thank R. Narasimhan for originally discussing this problem with me and for several useful conversations.

\section{REFERENCES}

1. W. L. Baily, Jr. and A. Borel, Compactification of arithmetic quotients of bounded symmetric domains, Ann. of Math. (2) 84 (1966), 442-528.

2. E. Calabi, Métriques Kählériennes et fibrés holomorphes, Ann. Sci. École Norm. Sup. (4) 12 (1979), 269-294.

3. S. Y. Cheng and S.-T. Yau, On the existence of a complete Kähler metric on non-compact complex manifolds and the regularity of Fefferman's equation, Comm. Pure Appl. Math. 33 (1980), 507-544.

4. H. Grauert, Bemerkenswerte pseudokonvexe Mannigfaltigkeiten, Math. Z. 81 (1963), 377-391.

5. N. Mok, Compactification of complete Kähler surfaces of finite volume satisfying certain curvature conditions, Ann. of Math. (2) 129 (1989), 383-425.

6. N. Mok and S.-T. Yau, Completeness of the Kähler-Einstein metric on bounded Riemann domains and the characterization of domains of holomorphy by curvature conditions, Proc. Sympos. Pure Math., vol. 39, part 1, Amer. Math. Soc., Providence, RI, 1983, pp. 41-60.

7. N. Mok and J.-Q. Zhong, Compactifying complete Kähler-Einstein manifolds of finite topological type and bounded curvature, Ann. of Math. (2) 129 (1989), 427-470.

8. A. Nadel, On complex manifolds which can be compactified by adding finitely many points, Invent. Math. 101 (1990), 173-189.

9. A. Nadel and H. Tsuji, Compactification of complete Kähler manifolds of negative curvature, J. Differential Geom. 28 (1988), 503-512.

10. T. Napier, Convexity properties of coverings of smooth projective varieties, Math. Ann. 286 (1989), 433-479.

11. R. Narasimhan, The Levi problem for complex spaces, Math. Ann. 142 (1961), 355-365.

12. T. Ohsawa, On complete Kähler domains with $C^{1}$-boundary, Publ. Res. Inst. Math. Sci. 16 (1980), 929-940.

13. Y.-T. Siu and S.-T. Yau, Compactification of negatively curved complete Kähler manifolds of finite volume, Ann. of Math. Stud., vol. 102, Princeton Univ. Press, Princeton, NJ, 1982, pp. 363-380.

14. A. Takeuchi, Domaines pseudoconvexes sur les variétés Kählériennes, J. Math. Kyoto Univ. 6 (1967), 323-357.

15. S.-T. Yau, Harmonic functions on complete Riemannian manifolds, Comm. Pure Appl. Math. 28 (1975), 201-228.

16. S.-K. Yeung, Compactification of complete Kähler surfaces with negative Ricci curvature, Invent. Math. 99 (1990), 145-163.

Department of Mathematics, University of Kentûcky, LeXington, Kentucky 405060027

E-mail address: pinney@ms.uky.edu 